\title{
INJECTIVE HULLS OF CERTAIN S-SYSTEMS OVER A SEMILATTICE
}

\author{
C. S. JOHNSON, JR. AND F. R. McMORRIS
}

\begin{abstract}
We construct, in the category of $S$-systems over a semilattice, the injective hulls of $S$-systems which are homomorphic images of $S$-subsystems of $S$.
\end{abstract}

1. Introduction. In [1] Berthiaume showed that injective hulls exist in the category of $S$-systems (or $S$-sets) over a semigroup $S$. In that paper he also showed that if $S$ is a chain then the injective hull of $S$ itself is its Dedekind-MacNeile completion. In the present paper we consider the case where $S$ is a semilattice and construct the injective hulls of $S$-systems which are homomorphic images of $S$-subsystems of $S$ (or, in the notation of [3], $S$-systems which are in $\boldsymbol{H S}(S)$ ). We do this by adapting the techniques used by Bruns and Lakser in [2] to construct injective hulls in the category of semilattices. We obtain as corollaries Berthiaume's result for chains, a characterization of injective cyclic $S$-systems over a semilattice, and the result that a semilattice $S$ is injective in the category of semilattices if and only if it is injective in the category of $S$-systems.

2. Preliminaries. Let $S$ be a semigroup. A (right) $S$-system is a set $M$ equipped with a map (written multiplicatively) from $M \times S$ to $M$ such that $m\left(s_{1} s_{2}\right)=\left(m s_{1}\right) s_{2}$ for all $m \in M$ and all $s_{1}, s_{2} \in S$. If one thinks of each element of $S$ as inducing a unary operation on an $S$-system $M$, then $M$ is a finitary algebra and all the notions of universal algebra are available. Thus if $M$ and $N$ are $S$-systems we have $A \subseteq M$ is an $S$-subsystem of $M$ if and only if $A S \subseteq A, \phi: M \rightarrow N$ is a homomorphism if and only if $\phi(m s)=\phi(m) s$ for all $m \in M$ and all $s \in S$, and an equivalence relation $\sim$ on $M$ is a congruence relation if and only if $m_{1} \sim m_{2}$ implies $m_{1} s \sim m_{2} s$ for all $s \in S$. Unless otherwise stated, all algebraic notions will be in this category. We will assume throughout that the semigroup $S$ is a semilattice (i.e., commutative and idempotent).

LeMma 1. If an $S$-system $M$ has the property that $M S=M$, then it is partially ordered by the rule $m_{1} \leqq m_{2}$ if and only if $m_{1}=m_{2}$ s for some $s \in S$.

Presented to the Society, June 1, 1971; received by the editors June 1, 1971. AMS 1970 subject classifications. Primary 20M99; Secondary 16A52, $18 \mathrm{~B} 99$. $K e y$ words and phrases. $S$-systems, injective $S$-systems. 
Proof. For each $m \in M$ we have $m=m_{1} s$ for some $m_{1} \in M$ and $s \in S$ (since $M S=M$ ), and hence $m=m s$, so $m \leqq m$. If $m_{1} \leqq m_{2}$ and $m_{2} \leqq m_{1}$ we have $s_{1}, s_{2} \in S$ such that $m_{1}=m_{2} s_{1}$ and $m_{2}=m_{1} s_{2}$. Now $m_{1}=m_{2} s_{1}=\left(m_{1} s_{2}\right) s_{1}=$ $m_{1}\left(s_{2} s_{1}\right)=\left(m_{2} s_{1}\right)\left(s_{2} s_{1}\right)=m_{2}\left(s_{1} s_{2} s_{1}\right)=m_{2}\left(s_{1} s_{2}\right)=\left(m_{2} s_{1}\right) s_{2}=m_{1} s_{2}=m_{2}$. Transitivity is obvious.

Notice that if $S$ has an identity and $M$ is a unitary $S$-system, then $M S=$ $M$ and Lemma 1 applies.

When we are dealing with a partial order on an $S$-system we will use the symbols " $\vee$ " and " $\wedge$ " to denote least upper bounds and greatest lower bounds, respectively.

We will refer to the partial order of Lemma 1 as the natural partial order on $M$.

If an $S$-system $M$ is partially ordered in some way and if $A \subseteq M$ is such that $\bigvee A$ exists, we will say that $\bigvee A$ is $S$-distributive if and only if, for each $s \in S, \bigvee\{a s \mid a \in A\}$ exists and equals $(\bigvee A) s$.

Recall the following definitions in a category of algebras: An algebra $C$ is injective if and only if every homomorphism from a subalgebra $A$ of an algebra $B$ into $C$ has an extension to all of $B$. An extension $C$ of an algebra $A$ is essential if and only if any homomorphism from $C$ to an algebra $B$, whose restriction to $A$ is one-to-one, is itself one-to-one. An injective hull of an algebra is an essential, injective extension.

LEMMA 2. Let $C$ be an $S$-system which is partially ordered in such a way that $c=\bigvee\{c s \mid s \in S\}$ for each $c \in C$. If $C$ is a complete lattice in which arbitrary joins are $S$-distributive, then $C$ is injective.

Proof. Let $A$ be an $S$-subsystem of an $S$-system $B$ and let $\phi: A \rightarrow C$ be a homomorphism. Define $\phi^{*}: B \rightarrow C$ by

$$
\phi^{*}(b)=\bigvee\{\phi(a) \mid a \in A, a=b s \text { for some } s \in S\} .
$$

If $b \in A$, then

$$
\phi^{*}(b)=\bigvee\{\phi(b s) \mid s \in S\}=\bigvee\{\phi(b) s \mid s \in S\}=\phi(b)
$$

and thus $\phi^{*}$ extends $\phi$. If $s_{0} \in S$ it is easy to see that $\left\{a s_{0} \mid a \in A, a=b s\right.$ for some $s \in S\}=\left\{a \mid a \in A, a=b s_{0} s\right.$ for some $\left.s \in S\right\}$. Thus

$$
\begin{aligned}
\phi^{*}(b) s_{0} & =(\bigvee\{\phi(a) \mid a \in A, a=b s \text { for some } s \in S\}) s_{0} \\
& =\bigvee\left\{\phi(a) s_{0} \mid a \in A, a=b s \text { for some } s \in S\right\} \\
& =\bigvee\left\{\phi\left(a s_{0}\right) \mid a \in A, a=b s \text { for some } s \in S\right\} \\
& =\bigvee\left\{\phi(a) \mid a \in A, a=b s_{0} s \text { for some } s \in S\right\}=\phi^{*}\left(b s_{0}\right) .
\end{aligned}
$$

We will call a subset $A$ of a poset $C$ join-dense in $C$ if and only if $c=$ $\bigvee\{a \in A \mid a \leqq c\}$ for each $c \in C$. If $A$ and $C$ are also $S$-systems we will say that $S$-distributive joins in $A$ are preserved in $C$ if and only if $a=\bigvee_{C} B$ 
whenever $B \subseteq A$ and $a=\bigvee_{A} B$ is $S$-distributive. We will call a map $\phi$ on a poset $P$ decreasing if and only if $\phi(a) \leqq a$ for all $a \in P$.

LEMMA 3. Let $C$ be an $S$-system which is partially ordered in such a way that the unary operations induced by $S$ preserve the order and are decreasing. Let $A$ be an S-subsystem of $C$ and suppose that for each $a \in A$ there is an $s_{a} \in S$ such that, for each $c \in C, c \wedge a$ exists and equals $c s_{a}$. If $A$ is join-dense in $C$ and if $S$-distributive joins in $A$ are preserved in $C$, then $C$ is an essential extension of $A$.

Proof. Let $\phi: B \rightarrow C$ be a homomorphism with $\left.\phi\right|_{A}$ one-to-one. If $\phi$ is not one-to-one there exist elements $a, b \in C$ with $a \neq b$ and $\phi(a)=\phi(b)$. Since $A$ is join-dense in $C$ we may suppose there exists $u \in A$ with $u \leqq b$ and $u$ a. We have $\phi(a \wedge u)=\phi\left(a s_{u}\right)=\phi(a) s_{u}=\phi(b) s_{u}=\phi\left(b s_{u}\right)=\phi(b \wedge u)=\phi(u)$. Now suppose $s \in S$ and let $M=\{(u \wedge x) s \mid x \leqq a, x \in A\}$. If we show that $u s=\bigvee_{A} M$ we will have shown (considering the special case $s=s_{u}$ ) that $u=\bigvee_{A}\{u \wedge x \mid x \leqq a, x \in A\}$ and is an $S$-distributive join. Hence $u=$ $\bigvee_{C}\{u \wedge x \mid x \leqq a, x \in A\} \leqq a$, a contradiction. Since $u \wedge x \leqq u$ implies $(u \wedge x) s \leqq$ $u s$, it is clear that $u s$ is an upper bound for $M$. Let $v \in A$ be another upper bound for $M$ with $v \neq u s$. Since meets exist in $A$ we may further assume that $v<u s$. If $c \in A$ and $c \leqq(u \wedge a) s$ we have $c \leqq u s$ and $c=u s \wedge c=u s s_{c}=u s_{c} s=$ $(u \wedge c) s$ with $c \leqq a s \leqq a$. Hence we can again use the fact that $A$ is join-dense in $C$ and obtain

$$
(u \wedge a) s=\bigvee_{C}\{(u \wedge x) s \mid x \leqq a, x \in A\}=\bigvee_{C} M \leqq v
$$

Now we have

$$
\begin{aligned}
\phi(u s) & =\phi(u) s=\phi(a \wedge u) s=\phi((a \wedge u) s)=\phi((a \wedge u) s \wedge v)=\phi\left((a \wedge u) s s_{v}\right) \\
& =\phi(a \wedge u) s s_{v}=\phi(u) s s_{v}=\phi\left(u s s_{v}\right)=\phi(u s \wedge v)=\phi(v)
\end{aligned}
$$

a contradiction. This establishes the fact that $u s=\bigvee_{A} M$ and finishes the proof.

3. Injective hulls. Let $M$ be an $S$-system such that $M S=M$. Recall that, by Lemma 1, $M$ is partially ordered by the rule $m_{1} \leqq m_{2}$ if and only if $m_{1}=m_{2} s$ for some $s \in S$. Following Bruns and Lakser we will call a subset $N$ of $M$ admissible if and only if $\bigvee N$ exists and is $S$-distributive, and we will call $N$ a $D$-ideal if and only if $y \in N$ and $x \leqq y$ imply $x \in N$ (i.e., $N S \subseteq N$ ) and $N$ is closed under $S$-distributive joins (i.e., $A \subseteq N$ and $A$ admissible implies $\bigvee A \in N)$. Now $I_{D}(M)$, the set of all $D$-ideals of $M$, is closed under arbitrary intersections and is thus a complete lattice under set inclusion. An obvious modification of the proof of [2, Lemma 3] shows that the join operation in $I_{D}(M)$ is given by

$$
\bigvee\left\{A_{i} \mid i \in I\right\}=\left\{\bigvee N \mid N \subseteq \bigcup\left\{A_{i} \mid i \in I\right\}, N \text { admissible }\right\}
$$


It is easy to show that if $N$ is a $D$-ideal of $M$ then $N s=\{n s \mid s \in S\}$ is also a $D$-ideal and that $N s=N \cap M s$. Thus $I_{D}(M)$ is a complete lattice in which arbitrary joins are $S$-distributive. Notice that $m S=\{x \in M \mid x \leqq m\}$, that these principal ideals are clearly $D$-ideals and that $m \mapsto m S$ is an embedding of $M$ in $I_{D}(M)$. Now, considering $M$ as an $S$-subsystem of $I_{D}(M)$, notice that $S$-distributive joins in $M$ are preserved in $I_{D}(M)$.

It is clear that $S$ itself is an $S$-system and we now restrict our attention to $\boldsymbol{H} \boldsymbol{S}(S)$, that is, to $S$-systems which are of the form $A / \sim$ where $A$ is an $S$ subsystem of $S$ and $\sim$ is a congruence relation on $A$. Notice that $A$ is an ideal of $S$ and $\sim$ is a semigroup congruence on $A$ (since we have assumed $S$ to be commutative) and thus $A / \sim$ is a semilattice as well as an $S$-system. It is easy to see that $(A / \sim) S=A / \sim$ and that the partial order on $A / \sim$ as a semilattice coincides with the natural partial order of Lemma 1.

THEOREM. If $M \in H S(S)$, then $I_{D}(M)$ is the injective hull of $M$.

Proof. $M=A / \sim$ where $A \subseteq S$ is an ideal and $\sim$ is a congruence relation on $A$. Denoting arbitrary elements of $A / \sim$ by $[x]$ with $x \in A$, we have that $[a] S=M a$ since $[a] s=[a s]=[a s a]=[a s] a$ and $[x] a=[x a]=[a x]=$ $[a] x$. Since a $D$-ideal $N$ is the join of the principal ideals it contains we have

$$
\begin{aligned}
N & =\bigvee\{[a] S \mid[a] \in N\}=\bigvee\{N \cap M a \mid[a] \in N\} \\
& \subseteq \bigvee\{N \cap M s \mid s \in S\}=\bigvee\{N s \mid s \in S\} \subseteq N .
\end{aligned}
$$

Thus $N=\bigvee\{N s \mid s \in S\}$ for each $N \in I_{D}(M)$ so the hypotheses of Lemma 2 are satisfied and $I_{D}(M)$ is injective. Since the unary operations in $I_{D}(M)$ are given by $N s=N \cap M s$, for each $s \in S$, it is apparent that they preserve the order and are decreasing and that for each $[a] \in M$ we have $N a=N \cap M a=$ $N \cap[a] S$. Thus, by identifying $M$ with the $S$-subsystem of $I_{D}(M)$ consisting of the principal order ideals of $M$, we see that the hypotheses of Lemma 3 are satisfied and that $I_{D}(M)$ is an essential extension of $M$.

COROllary 1. If $\boldsymbol{M} \in \boldsymbol{H} \boldsymbol{S}(S)$, then $M$ is injective if and only if it is a complete lattice in which arbitrary joins are $S$-distributive.

Proof. $\quad M$ is injective if and only if the embedding $m \mapsto m S$ of $M$ in $I_{D}(M)$ is onto. This is true precisely when every $D$-ideal of $M$ is principal. Clearly this is the case when $M$ is a complete lattice in which arbitrary joins are $S$-distributive. Conversely, if every $D$-ideal is principal, then the partial ordering of $I_{D}(M)$ by set inclusion (under which $I_{D}(M)$ is a complete lattice with $S$-distributive joins) coincides with its natural partial order as an $S$-system, i.e., $m_{1} S \subseteq m_{2} S$ if and only if $m_{1}=m_{2} s$ for some $s \in S$. Since in this case $M$ is isomorphic to $I_{D}(M), M$ is also a complete lattice with $S$-distributive joins. 
Corollary 2 (Berthiaume). If $S$ is a chain, then its injective hull is its Dedekind-MacNeile completion.

Proof. If $S$ is a chain, then every order ideal is a $D$-ideal and hence $I_{D}(S)$ is the Dedekind-MacNeile completion.

COROLlary 3. A semilattice $S$ is injective in the category of semilattices if and only if it is injective in the category of $S$-systems.

Proof. By Corollary $1, S$ is injective in the category of $S$-systems if and only if it is a complete lattice with the property that $(\bigvee M) \wedge s=$ $\bigvee\{m \wedge s \mid m \in M\}$ for all $s \in S, M \subseteq S$. By [2, Theorem 1] these properties characterize injectivity in the category of semilattices.

COROLlary 4. A cyclic $S$-system is injective if and only if it is a complete lattice (in its natural partial order) in which arbitrary joins are $S$-distributive.

Proof. If $M$ is a cyclic $S$-system, then $M=x S$ for some $x \in M$. It is clear that $M S=M$, so $M$ has a natural partial order (Lemma 1). Define a congruence relation on $S$ by $s_{1} \sim s_{2}$ if and only $x s_{1}=x s_{2}$. The map $x s \mapsto[s]$ is an isomorphism between $M$ and $S / \sim$ and hence $M \in H S(S)$ and Corollary 1 applies.

COROLlaRY 5. Let $M$ be an $S$-system such that $M S=M$. Iffor each $m \in M$ there exists an $s \in S$ such that $m S=M s$, then $M$ is injective if and only if it is a complete lattice in which arbitrary joins are $S$-distributive.

Proof. Define a congruence relation on $S$ by $s_{1} \sim s_{2}$ if and only if $M s_{1}=M s_{2}$. The map $m \mapsto[s]$, where $m S=M s$, is an isomorphism between $M$ and an $S$-subsystem of $S / \sim$. Since $\boldsymbol{S H}(S) \subseteq \boldsymbol{H S}(S)$ by [3, Theorem 1, p. 152], $\boldsymbol{M \in H S}(S)$ and Corollary 1 applies.

\section{REFERENCES}

1. P. Berthiaume, The injective envelope of $S$-sets, Canad. Math. Bull. 10 (1967), 261273. MR 35 \#4321.

2. G. Bruns and H. Lakser, Injective hulls of semilattices, Canad. Math. Bull. 13 (1970), 115-118.

3. G. Grätzer, Universal algebra, Van Nostrand, Princeton, N.J., 1968. MR 40 \#1320.

Department of Mathematics, Bowling Green State University, Bowling Green, OHio 43403

Current address (McMorris): Biomathematics Program, Box 5457, North Carolina State University, Raleigh, North Carolina 27607 